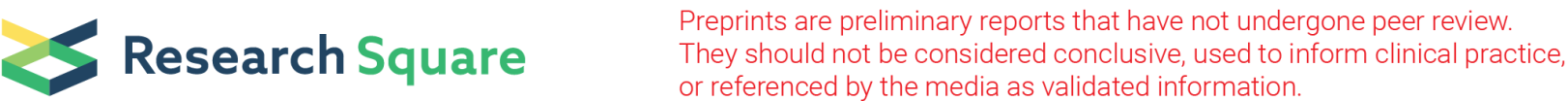

\section{The Socioeconomic Burden of Pneumonia Due to Multidrug-Resistant Acinetobacter Baumannii and Pseudomonas Aeruginosa in Korea: A Prospective Case-Control Study}

\section{Chung-Jong Kim}

Ewha Womans University Medical Center

Kyoung-Ho Song

Seoul National University Bundang Hospital

Nam-Kyong Choi

Ewha Womans University

Jeonghoon Ahn

Ewha Womans University

Ji Yun Bae

Ewha Womans University Medical Center

Hee Jung Choi

Ewha Womans University Medical Center

Younghee Jung

Hallym University Sacred Heart Hospital

Seung Soon Lee

Hallym University Sacred Heart Hospital

Ji-Hwan Bang

Boramae Medical Center

\section{Eu Suk Kim}

Seoul National University Bundang Hospital

\section{Song Mi Moon}

Seoul National University Bundang Hospital

Je Eun Song

Inje University Ilsan Paik Hospital

Yee Gyung Kwak

Inje University Ilsan Paik Hospital

Shin Hye Chun

Chungnam National University Hospital

Yeon-Sook Kim

Chungnam National University Hospital 


\section{Kyung-Hwa Park}

Chonnam National University Hospital

\section{Yu Min Kang}

Kangwon National University Hospital

\section{Pyoeng Gyun Choe}

Seoul National University Hospital

\section{Shinwon Lee}

Pusan National University Hospital

Hong Bin Kim ( $\square$ hbkimmd@snu.ac.kr)

Seoul National University Bundang Hospital

Korea INfectious Diseases study Group (KIND)

KIND Study Group

\section{Research Article}

Keywords: Acinetobacter baumannii, Pseudomonas aeruginosa, pneumonia, Disease burden, Multi-drug resistant

Posted Date: January 17th, 2022

DOI: https://doi.org/10.21203/rs.3.rs-1223775/v1

License: (c) (i) This work is licensed under a Creative Commons Attribution 4.0 International License. Read Full License 


\section{Abstract}

We aimed to estimate the socioeconomic burden of pneumonia due to multidrug-resistant Acinetobacter baumannii (MRAB) and Pseudomonas aeruginosa (MRPA). We prospectively searched for MRAB and MRPA pneumonia cases and matched them with controls with susceptible-organism pneumonia and non-infected cases from ten hospitals over a 6-month period. The matching criteria were: same principal diagnosis, same surgery or intervention during hospitalization, age (years \pm 10 ), sex, and admission date within 60 days. The total additional direct medical cost was estimated by the multistate model. A total of 108 cases of MRAB pneumonia [MRAB-P] and 28 cases of MRPA pneumonia [MRPA-P] were identified. The 90 -day mortality rate was $37.0 \%$ and $39.3 \%$ for MRAB-P and MRPA-P, respectively. The additional medical costs were $\$ 42,203$ and $\$ 35,556$ for MRAB-P and MRPA-P, respectively. The estimated number of MRAB-P and MRPA-P cases were 1,309-2,483 and 339-644, respectively with estimated deaths being 485-920 and 133-253 in a year, respectively. The annual socioeconomic burden was $\$ 12,053,593-$ $\$ 22,898,271$ and $\$ 15,241,883-\$ 28,994,008$, respectively. We estimated that MRAB-P and MRPA-P occurred in $1,648-3,172$ patients, with $618-1,173$ deaths, and caused $\$ 27,295,476-\$ 51,892,279$ in socioeconomic burden nationwide. It is time to investigate and invest in prevention of multidrug-resistant bacterial pneumonia.

\section{Introduction}

Pneumonia is a complex disease with varied aetiology. Most studies on the burden of disease caused by pneumonia to date focussed on community acquired pneumonia (CAP) or pneumonia in paediatric populations ${ }^{1-8}$. However, little is known about the burden of disease for pneumonia occurring in hospitals, particularly pneumonia due to multidrug resistant organisms ${ }^{9}$.

In a meta-analysis, the incidence of nosocomial pneumonia was $12.8-20.4 \%$, and that of ventilator associated pneumonia (VAP) was 31.4-36.1\% ${ }^{9}$. Another study reported an incidence of VAP of 18.3 episodes per 1,000 ventilator days ${ }^{10}$. The mortality rate of nosocomial pneumonia is $21-37.4 \%{ }^{11}$, and the occurrence of nosocomial pneumonia extends hospital stay by 18.0-30 days ${ }^{9}$. Klaus Kaier et al., reported that nosocomial Pseudomonas aeruginosa pneumonia incurs an additional medical cost of $€ 19,000^{12}$.

The causative agent in nosocomial pneumonia is often a strain that is difficult to treat. Currently, known causative bacteria are mainly P. aeruginosa, Klebsiella pneumoniae, Staphylococcus aureus, and Acinetobacter baumannii, ${ }^{13}$ and, unlike CAP, causative agents are often antibiotic-resistant bacteria. Infection due to multidrug-resistant (MDR) A. baumannii (MRAB) or $P$. aeruginosa (MRPA) are more difficult to treat, and antibiotics with a relatively high frequency of adverse events, such as colistin, are necessary in many cases. Accordingly, pneumonia caused by multidrug-resistant organisms (MDRO) are likely to have a very high socioeconomic burden. 
To date, studies reporting on the disease burden of pneumonia caused by MDRO are scarce. In this study, we described the clinical characteristics and estimated the disease burden of pneumonia due to MDR $A$. baumannii and $P$. aeruginosa on a nationwide scale.

\section{Results}

\section{Clinical characteristics}

During the 6-month study period, 136 cases of MDRO pneumonia were detected (108 cases of MRAB pneumonia [MRAB-P] and 28 cases of MRPA pneumonia [MRPA-P]).

The mean age of patients with MRAB-P and MRPA-P was 69.4 years $( \pm 16.8$ years) and 67.0 years $( \pm 7.1$ years), respectively. Both types of bacteria were more common in male patients (MRAB-P $65.7 \%$, MRPA-P $57.1 \%)$. MRAB-P occurred in the ICU and the ward in $63 \%$ and $35 \%$ of cases, respectively, and MRPA-P in $46.4 \%$ and $50 \%$, respectively. Hospital onset infection accounted for $94 \%$ of MRAB-P, and $71.4 \%$ of MRPAP cases (Table 1). 
Table 1

Clinical characteristics and outcomes of enrolled patients with pneumonia due to multidrug-resistant Acinetobacter baumannii and Pseudomonas aeruginosa for a six-month period from ten hospitals in Korea.

\begin{tabular}{|c|c|c|c|}
\hline & $\begin{array}{l}\text { MRAB-P } \\
n=108\end{array}$ & $\begin{array}{l}\text { MRPA-P } \\
n=28\end{array}$ & p-value \\
\hline Age & $69.4( \pm 16.8)$ & $67.0( \pm 17.1)$ & 0.508 \\
\hline Sex (male) & $71(65.7 \%)$ & $16(57.1 \%)$ & 0.398 \\
\hline \multicolumn{4}{|l|}{ Location } \\
\hline Ward & $38(35.2 \%)$ & $14(50.0 \%)$ & \\
\hline Intensive care unit & $68(63.0 \%)$ & $13(46.4 \%)$ & \\
\hline Emergency room & $2(1.9 \%)$ & $1(3.6 \%)$ & \\
\hline Out-patient clinic & 0 & 0 & \\
\hline LOS (median, (IQR)) & $59(37-85)$ & $45(36-102)$ & 0.486 \\
\hline Post bacteraemia LOS (median, (IQR)) & $24(9-46)$ & $28(10-59)$ & 0.792 \\
\hline SOFA score (median, (IQR)) & $5(2-8)$ & $3(1-7)$ & 0.138 \\
\hline \multicolumn{4}{|l|}{ Mortality } \\
\hline In hospital & $42(38.9 \%)$ & $10(35.7 \%)$ & 0.830 \\
\hline 7 days & $4(3.7 \%)$ & $4(14.3 \%)$ & 0.056 \\
\hline 30 days & $26(24.1 \%)$ & $9(32.1 \%)$ & 0.467 \\
\hline 90 days & $40(37.0 \%)$ & 11 (39.3\%) & 0.830 \\
\hline
\end{tabular}

Patients with MRAB-P had a mean LOS of 73.2 days ( \pm 55.5 days), with the mean LOS before and after the onset of pneumonia being 19.9 days ( \pm 22.8 days) and 39.9 days ( \pm 45.0 days), respectively. The mean LOS for patients with MRPA-P was 92.6 days ( \pm 153.5 days), with the LOS before and after the onset of pneumonia being 44.4 days ( \pm 103.5 days) and 39.1 days ( \pm 37.6 days), respectively.

There were 34 (31.5\%) and five patients (17.8\%) with severe sepsis or shock among MRAB-P and MRPA-P patients, respectively. The 30-day, and 90 -day, mortality rates were $24.1 \%$ and $37.0 \%$ for MRAB-P, and $32.1 \%$ and $39.3 \%$ for MRPA-P, respectively, with no significant difference between the two groups $(p=0.384, p=0.827)$. The 90 -day mortality rate by age is shown in the web-only Supplementary Table $S 1$. 


\section{Matching Of Control Patients}

Fifty-four cases of pneumonia with non-MDR A. baumannii and 81 cases of non-infection were matched with MRAB-P cases. Twenty-two cases of pneumonia with non-MDR P. aeruginosa and 18 cases of noninfection were matched with MRPA-P cases. Six patients with MRAB-P and two patients with MRPA-P were excluded because of a LOS longer than 180 days.

Additional hospital cost and LOS: MDRO vs susceptible organism infection or no infection

The mean difference in LOS between the R-group and S-group MRAB-P patients was 19 days $( \pm 50.5$ days), and in hospital cost was $\$ 18,833( \pm \$ 33,236)$. The difference in the LOS between R-group and Sgroup MRPA-P patients was 14 days $( \pm 45.8$ days), and in hospital cost was $\$ 12,250( \pm \$ 33,447)$ (Table 2$)$.

Table 2

Differences in costs and lengths of hospital stay between patients with multidrug-resistant (MDR) and non-MDR bacterial pneumonia

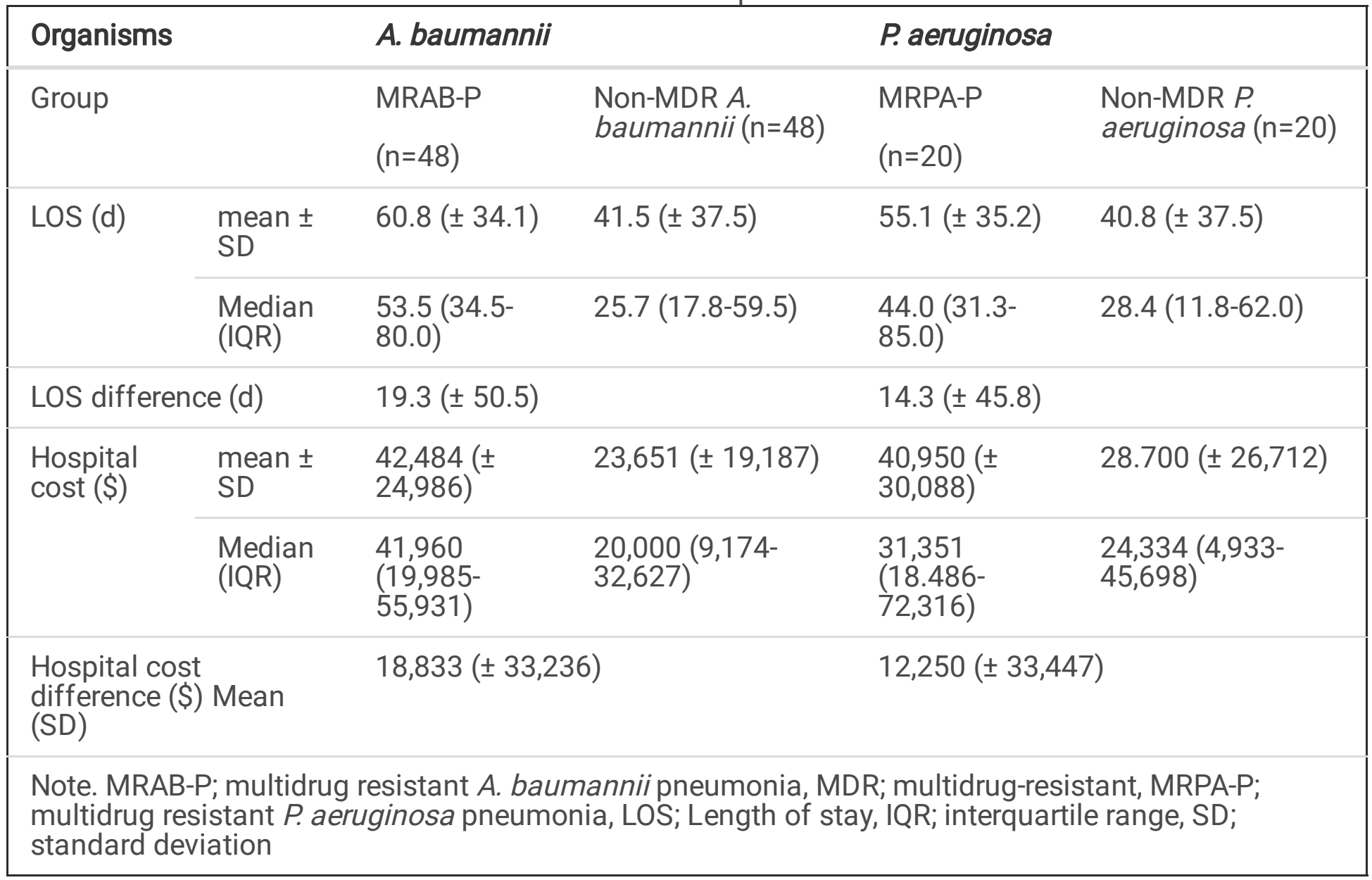

The mean difference in LOS between the R-group and N-group MRAB-P patients was 48.5 days $( \pm 42.0$ days), and the difference in hospital cost was $\$ 42,203$.

The mean difference in LOS between the R-group and N-group MRPA-P patients was 44.7 days $( \pm 38.3$ days), and the difference in hospital cost was $\$ 35,556$ (Table 3). 
Table 3

Differences in costs and length of hospital stay between patients with multidrug resistant organisms' pneumonia and no infection

\begin{tabular}{|c|c|c|c|c|c|}
\hline \multicolumn{2}{|l|}{ Organisms } & \multicolumn{2}{|l|}{ A. baumannii } & \multicolumn{2}{|l|}{$P$. aeruginosa } \\
\hline \multirow{2}{*}{\multicolumn{2}{|c|}{ Group }} & MRAB-P & Non-infection & MRPA-P & Non-infection \\
\hline & & $(n=/ 5)$ & $(n=75)$ & $(n=1 /)$ & $(n=17)$ \\
\hline \multirow[t]{2}{*}{$\operatorname{LOS}(\mathrm{d})$} & $\begin{array}{l}\text { mean } \pm \\
\text { SD }\end{array}$ & $60.1( \pm 36.0)$ & $11.7( \pm 18.6)$ & $56.5( \pm 37.8)$ & $11.8( \pm 6.5)$ \\
\hline & $\begin{array}{l}\text { Median } \\
\text { (IQR) }\end{array}$ & $50.0(33.0-80.0)$ & $7.3(5.5-12.3)$ & $44.0(27.5-97.0)$ & $9.2(6.3-15.4)$ \\
\hline \multicolumn{2}{|c|}{ LOS difference (d) } & \multicolumn{2}{|l|}{$48.5( \pm 42.0)$} & \multicolumn{2}{|l|}{$44.7( \pm 38.3)$} \\
\hline \multirow[t]{2}{*}{$\begin{array}{l}\text { Hospital cost } \\
\text { (\$) }\end{array}$} & $\begin{array}{l}\text { mean } \pm \\
\text { SD }\end{array}$ & $\begin{array}{l}50,403( \pm \\
33,090)\end{array}$ & $\begin{array}{l}8,200( \pm \\
15,331)\end{array}$ & $\begin{array}{l}43,336( \pm \\
32,091)\end{array}$ & $\begin{array}{l}7,780( \pm \\
4,318)\end{array}$ \\
\hline & $\begin{array}{l}\text { Median } \\
\text { (IQR) }\end{array}$ & $\begin{array}{l}47,616(24,351- \\
64,812)\end{array}$ & $\begin{array}{l}4,260(2,933- \\
8,070)\end{array}$ & $\begin{array}{l}32,187(14,828- \\
77,191)\end{array}$ & $\begin{array}{l}6,356(4,681- \\
11,341)\end{array}$ \\
\hline \multicolumn{2}{|c|}{$\begin{array}{l}\text { Hospital cost difference } \\
\text { (\$) Mean (SD) }\end{array}$} & \multicolumn{2}{|l|}{$42,203( \pm 35,792)$} & \multicolumn{2}{|l|}{$35,556( \pm 31,834)$} \\
\hline
\end{tabular}

\section{Cost of caregiver}

In patients with MRAB-P, the additional caregiver cost was $\$ 1,113$, for 19 days of extended hospital stay compared to those with susceptible pneumonia. The non-infected control group hospital stay was extended by 49 days, with a caregiver cost of $\$ 2,869$.

MRPA-P patients had an extension of hospital stay of 14 days compared to those with pneumonia caused by susceptible bacteria, with an additional caregiver cost of $\$ 820$. In the non-infected control group, a caregiver cost of $\$ 2,635$ was added.

\section{Estimation of nationwide disease burden: number of cases and productivity loss due to unexpected death}

In the MDR bacteraemia study conducted together with this study, the number of patients with bacteraemia in 10 hospitals was $8.7-16.5 \%$ of the estimated number of patients nationwide (detailed estimation methods are included in the web-only supplementary results and Supplementary Table S2). Assuming that the incidence rate of MDRO pneumonia is similar, the estimated number of MRAB-P patients in South Korea is a minimum of 1309 to a maximum of 2483 , and that of MRPA-P is a minimum of 339 to a maximum of 644 (see web-only Supplementary Table S1). 
The number of deaths from MRAB-P and MRPA-P was 485-920 and 133-253 patients, respectively, calculated with the 90-day and age-specific mortality rates obtained in the study.

\section{Economic Burden Of Pneumonia Due To Mdro Infection}

The total additional hospital costs due to MRAB-P was $\$ 55,243,476-\$ 104,789,573$ compared to the noinfection group. When the cost of the caregiver and the productivity loss caused by death were added, the economic burden caused by MRAB-P was $\$ 64,549,723-\$ 122,533,585$.

The total additional hospital costs due to MRPA-P was $\$ 12,053,593-\$ 22,898,271$ compared to the susceptible group. When the cost of nursing care and social loss due to death were added, the economic loss caused by MRPA-P was $\$ 15,241,883-\$ 28,994,008$ (Table 4).

Table 4

Results of socioeconomic burden estimation of two multidrug-resistant organisms' pneumonia

\begin{tabular}{|c|c|c|c|c|}
\hline & MRAB-P & & MRPA-P & \\
\hline & minimum & maximum & minimum & maximum \\
\hline Number of cases in $2017(\mathrm{~N})$ & 1309 & 2483 & 339 & 644 \\
\hline Hospital cost differences (C) (\$) & 42,203 & & 35,556 & \\
\hline LOS differences (L) (d) & 49 & & 45 & \\
\hline 90 day mortality rate & $37.0 \%$ & $37.0 \%$ & $39.3 \%$ & $39.3 \%$ \\
\hline Total hospital cost (NXC) (\$) & $55,243,476$ & $104,789,573$ & $12,053,593$ & $22,898,271$ \\
\hline $\begin{array}{l}\text { Excess cost of caregiver use }(\$)(L X \\
58.56)\end{array}$ & $2,869.4$ & & $2,635.2$ & \\
\hline $\begin{array}{l}\text { Total cost of excess caregiver use }(\$)(L X \\
58.56 \times N)\end{array}$ & $3,756,005$ & $7,124,644$ & 893,333 & $1,697,069$ \\
\hline Estimated number of deaths in 1 year & 485 & 920 & 133 & 253 \\
\hline Productivity loss due to mortality ${ }^{\star}(\$)$ & $5,550,242$ & $10,619,368$ & $2,294,957$ & $4,398,668$ \\
\hline Total socioeconomic burden (\$) & $64,549,723$ & $122,533,585$ & $15,241,883$ & $28,994,008$ \\
\hline
\end{tabular}

\section{Discussion}

In this study, we estimated the disease burden of pneumonia caused by MRAB and MRPA, which are common causes of nosocomial pneumonia. The economic burden of MDRO pneumonia was $\$ 42,203$ 
and $\$ 35,556$ for MRAB-P and MRPA-P, respectively. The estimated number of MDRO pneumonia cases in South Korea for one year was 1,309-2,483 and 339-644 for MRAB-P and MRPA-P, respectively. Annual deaths due to MDRO pneumonia were estimated to be 485-920 and 133-253 in MRAB-P and MRPA-P. The socioeconomic burden of MRAB-P and MRPA-P was $\$ 55,243,476-\$ 104,789,573$ and $\$ 64,549,723-$ $\$ 122,533,585$, respectively.

In this study, the prognosis of MRAB-P and MRPA-P were similar to previous studies. The proportion of these two bacteria among the causative agents of nosocomial pneumonia and VAP was reported to be about $30-34 \%$ and $35.6 \%$, respectively 9,14 . The previously known mortality rate of Acinetobacter pneumonia was $37.2-48.1 \%{ }^{15}$, and Acinetobacter infection caused an additional economic burden of $\$ 6,693-\$ 16,074$. Mortality of MDR Pseudomonas pneumonia is also high at $34.6 \%{ }^{16}$, and the median length of ICU stay was 34 days. In previous systematic reviews, the overall mortality of ICU pneumonia was $37.4 \%$, and that of VAP was $34.5 \%{ }^{9}$. LOS in ICU pneumonia was 17.7 days, and that of VAP was 30.5 days ${ }^{9}$.

MRAB and MRPA are known to be the major causative agents of nosocomial pneumonia. Because nosocomial infections are unexpected, and in many cases, preventable, the socioeconomic burden is also unexpected and preventable. In a recent study, Andrew et al., reported that multifaceted prevention programs are cost-effective in nosocomial infection ${ }^{17}$. By acquiring a new disease irrelevant to the reason for admission to the hospital, additional medical resources are consumed and hospital stay is prolonged, leading to socioeconomic loss. Additionally, broad-spectrum antibiotics are usually needed for MDRO, which can also be a factor in the burden of additional disease. In this study, disease burden between MDRO infection and susceptible organism infection or no-infection were compared. Even if there was an infectious disease caused by the same bacteria, antibiotic resistance caused an additional burden. Hospital costs were 1.80 times, and 1.42 times higher than susceptible organism infection in MRAB-P and MRPA-P, and 6.14 times and 5.57 times higher than non-infected controls in MRAB-P and MRPA-P. Vasudevan et al., reported that the median hospital cost per day of resistant gram-negative bacterial infection in ICU was 1.5 times higher than non-infection ${ }^{18}$. This is similar to our results where hospital cost per day was 1.2 times higher compared to the non-infected control group ( $\$ 840$ vs $\$ 700$ in MRAB-P, and \$ 767 vs \$ 659 in MRPA-P).

This study has the following limitations. First, it is difficult to conclude that the difference in cost between the R-group and the $\mathrm{N}$-group is due only to the pneumonia because the control group selection criteria were not broad. The cost difference can be ascribed only to pneumonia if the same conditions prevailed in the patient and control groups. However, in practice, it is impossible to select a control group for these patient groups in such a manner. This is a limitation of the multistate model, and interpretation of the results must consider this.

Second, some cases with respiratory colonization rather than pneumonia may be included in the study since the definition of pneumonia was crude. The definition used was cases where bacteria grew in sputum and antibiotics were used against them. Because MRAB or MRPA can appear as colonized flora 
in the respiratory tract even without pneumonia, treatment was included in the definition. Because the clinical picture of infiltration on chest X-ray, fever, and abnormal blood test findings, etc. are present in many ICU patients, even if they are not diagnosed with pneumonia, we applied this simple definition.

In conclusion, MRAB-P and MRPA-P infected 1,648-3,172 patients, with $618-1,173$ deaths, and caused $\$ 27,295,476-\$ 51,892,279$ of socioeconomic burden. It is time to investigate and invest in the prevention of MDRO pneumonia to decrease the burden.

\section{Methods}

\section{Study design}

We prospectively collected cases of pneumonia due to $A$. baumannii and $P$. aeruginosa, and matched them with two control patients, one with pneumonia due to non-multidrug resistant $A$. baumannii or $P$. aeruginosa, and one with no infection. We used the multistate model utilised by Stewardson et al ${ }^{19}$. Enrolled patients were categorized into three states: MDRO infection (R-group), susceptible organism infection (S-group), and no-infection (N-group). We compared the clinical and economic aspects of the Rgroup and S-group, and the R-group and N-group, respectively, and estimated the additional burden of pneumonia due to MDRO, compared to that of susceptible organisms, and no infection. Our study conforms to the Consolidated Health Economic Evaluation Reporting Standards (CHEERS), and all methods were carried out in accordance with relevant guidelines and regulations.

\section{Setting}

We collected data from ten secondary and tertiary hospitals in South Korea, selected by considering the regional distribution of hospitals. The study was performed from September 2017 to February 2018. We used a currency exchange rate of 1110 Korean won / 1 US dollar for the calculations.

\section{Participants}

We prospectively identified and collected the data of all patients with pneumonia due to A. baumannii, and $P$. aeruginosa, regardless of antibiotic susceptibilities. After collection, the R-group and S-group were selected by pre-defined criteria. In brief, MDR was defined as Acinetobacter or Pseudomonas isolates resistant to at least three classes of antimicrobial agents, including carbapenem, aminoglycosides, and fluoroquinolones. Pneumonia was defined as a case in which bacteria grew in respiratory specimens such as sputum, transtracheal aspiration fluid, and bronchoscopy washings, and susceptible antibiotics were administered against the organisms. The criteria for selected matched susceptible or no-infection control cases were: same principal diagnosis at the time of admission, same major surgery or intervention during the hospitalization, age (years \pm 10 ), sex, and admission date within 60 days.

If the control cases of susceptible infection or no infection experienced invasive bacterial infection during the same hospital admission, then the control case was excluded, and another was selected. Cases with 
a total length of stay (LOS) of 180 days or longer were excluded from matching.

\section{Variables}

Data collection variables were baseline characteristics, route of admission, LOS before and after infection, and underlying disease. We also collected data on severity of infection through the Sequential Organ Failure Assessment (SOFA) score, and 90-day mortality. Hospital costs of patients in each group were collected.

\section{Statistical analysis}

\section{Estimation of additional hospital and caregiver costs of MDRO pneumonia}

We estimated the total additional direct medical costs of MDRO pneumonia (R-group) by subtracting the mean hospital cost of the S-group or N-group from that of the corresponding R-group.

The cost of caregiving was calculated by multiplying the daily cost of the hired caregiver and the excess length of stay (LOS) (caregiver fee was $\$ 59.1$ per day [65,000 Korean won] as taken from the caregiver association).

\section{Estimation of numbers and mortality of MDRO pneumonia cases nationwide}

The estimation methods are described in the online only supplementary methods. In brief, we calculated the ratio of cases of MDRO bacteraemia between the 10 study hospitals and the national survey which we undertook previously. We then assumed that the ratio of pneumonia in the 10 study hospitals to the nationwide results was the same as that of MDRO bacteraemia (unpublished data). The number of occurrences of pneumonia and the number of deaths nationwide were estimated, based on this ratio.

We estimated the mortality due to MDRO pneumonia according to age distribution. We estimated the mortality rate for each age group in the R-group patients, and then calculated the ratio of patients by age group among the total deaths. The estimated number of deaths of each age cohort of MDRO pneumonia on a nationwide scale was calculated by multiplying the estimated number of MDRO pneumonia cases and the 90-day mortality rate from our data.

\section{Estimation of productivity loss due to death}

The productivity loss due to unexpected death was calculated from the number of deaths associated with MDRO pneumonia and the annual mean wages reported by the Ministry of Labor in Korea (Labor Statistics of Korea, Ministry of Employment and Labor 2017; available from http://wage.go.kr/index.jsp). The productivity loss due to the unexpected death of a given patient was the sum of the annual wages up to the time that patient would have reached 65 years of age if he or she had not died. The annual discount rate was $5 \%$. 
The socioeconomic burden of MDRO pneumonia was estimated by the sum of the additional hospital cost, caregiver cost, and productivity loss due to unexpected death. The additional hospital and caregiver costs were calculated by multiplying the additional cost due to MDRO pneumonia and the estimated annual number of MDRO pneumonia patients.

\section{Ethical review}

This study was approved by the Institutional Review Board (IRB) of Seoul National University Bundang Hospital (IRB no. B-1804-463-105) and the IRBs of each participating hospital. Informed consent was waived by the following IRBs; Seoul National University Bundang Hospital, Ewha Womans University Mokdong Hospital, Hallym University Sacred Heart Hospital, Seoul Metropolitan Government-Seoul National University Boramae Medical Center, Inje University Ilsan Paik Hospital, Chungnam National University Hospital, Chonnam National University Hospital, Kangwon National University Hospital, Seoul National University Hospital, and Pusan National University Hospital.

\section{Declarations}

\section{Acknowledgement}

none

\section{Author's Contributions}

CJK, KHS, JA, HBK formulated the research question, NKC Conceived and designed the analysis, JA directed the study's methodological implementation and helped revise the manuscript, CJK, KHS, JYB, HJC, YJ, SSL, JHB, ESK, SMM, JES, YGK, SHC, YSK, KHP, YMK, PGC, SL collected the data, CJK, NKC performed analysis, CJK, KHS conducted the data analysis and drafted the manuscript, all authors critically revised the manuscript, and approved the final version of the manuscript

\section{Competing interests}

The authors declare that they have no relevant financial or non-financial competing interests.

\section{Funding}

This work was supported by the Research Program funded by the Korea Disease Control and Prevention Agency (2017E280301).

\section{Data Availability}

The datasets used and analysed during the current study are available from the corresponding author on reasonable request.

\section{Ethics approval}


This study was approved by the Institutional Review Board (IRB) of the following institutions;

Seoul National University Bundang Hospital

Ewha Womans University Mokdong Hospital

Hallym University Sacred Heart Hospital

Seoul Metropolitan Government-Seoul National University Boramae Medical Center

Inje University Ilsan Paik Hospital

Chungnam National University Hospital

Chonnam National University Hospital

Kangwon National University Hospital

Seoul National University Hospital

Pusan National University Hospital

Consent to participate

Written informed consent was waived by institutional review board of each hospital

Consent for publish

Not applicable

\section{Members of the KIND study group who also contributed to this work are:}

Sang Won Park ${ }^{6}$, Chan Mi Lee ${ }^{2}$, Sook-In Jung ${ }^{9}$, Seong Eun Kim ${ }^{9}$, Wan Beom Park ${ }^{3}$ 11, Nam Joong Kim ${ }^{3}$,

11 , Sun Hee Lee ${ }^{12}$, Hyunju Lee ${ }^{13}$, Jeong Su Park ${ }^{14}$, Young-Jun Kim ${ }^{15}$

13 Department of Paediatrics, Seoul National University Bundang Hospital, Seongnam, South Korea

14 Department of Laboratory Medicine, Seoul National University Bundang Hospital, Seongnam, South Korea

15 Department of Internal Medicine, Wonkwang University Hospital, Iksan, Republic of Korea

\section{References}

1. Peyrani, P., Mandell, L., Torres, A. \& Tillotson, G. S. The burden of community-acquired bacterial pneumonia in the era of antibiotic resistance. Expert Rev Respir Med 13, 139-152, 
doi:10.1080/17476348.2019.1562339 (2019).

2. Singh, V. The burden of pneumonia in children: an Asian perspective. Paediatr Respir Rev 6, 88-93, doi:10.1016/j.prrv.2005.03.002 (2005).

3. Li, A., Newall, A. T., Britt, H. \& Macintyre, C. R. The cost and disease burden of pneumonia in general practice in Australia. Vaccine 30, 830-831, doi:10.1016/j.vaccine.2011.11.103 (2012).

4. Heo, J. Y. \& Song, J. Y. Disease Burden and Etiologic Distribution of Community-Acquired Pneumonia in Adults: Evolving Epidemiology in the Era of Pneumococcal Conjugate Vaccines. Infect Chemother 50, 287-300, doi:10.3947/ic.2018.50.4.287 (2018).

5. Choi, M. J. et al. Disease burden of hospitalized community-acquired pneumonia in South Korea: Analysis based on age and underlying medical conditions. Medicine (Baltimore) 96, e8429, doi:10.1097/MD.0000000000008429 (2017).

6. Lee, J. Y., Yoo, C. G., Kim, H. J., Jung, K. S. \& Yoo, K. H. Disease burden of pneumonia in Korean adults aged over 50 years stratified by age and underlying diseases. Korean J Intern Med 29, 764773, doi:10.3904/kjim.2014.29.6.764 (2014).

7. Shi, T. et al. Global and Regional Burden of Hospital Admissions for Pneumonia in Older Adults: A Systematic Review and Meta-Analysis. J Infect Dis 222, S570-S576, doi:10.1093/infdis/jiz053 (2020).

8. Drijkoningen, J. J. \& Rohde, G. G. Pneumococcal infection in adults: burden of disease. Clin Microbiol Infect 20 Suppl 5, 45-51, doi:10.1111/1469-0691.12461 (2014).

9. Zhang, Y. et al. Disease burden of intensive care unit-acquired pneumonia in China: a systematic review and meta-analysis. Int J Infect Dis 29, 84-90, doi:10.1016/j.jijid.2014.05.030 (2014).

10. Koulenti, D., Tsigou, E. \& Rello, J. Nosocomial pneumonia in 27 ICUs in Europe: perspectives from the EU-VAP/CAP study. Eur J Clin Microbiol Infect Dis 36, 1999-2006, doi:10.1007/s10096-016-2703-z (2017).

11. Behnia, M., Logan, S. C., Fallen, L. \& Catalano, P. Nosocomial and ventilator-associated pneumonia in a community hospital intensive care unit: a retrospective review and analysis. BMC Res Notes 7, 232, doi:10.1186/1756-0500-7-232 (2014).

12. Kaier, K., Heister, T., Gotting, T., Wolkewitz, M. \& Mutters, N. T. Measuring the in-hospital costs of Pseudomonas aeruginosa pneumonia: methodology and results from a German teaching hospital. BMC Infect Dis 19, 1028, doi:10.1186/s12879-019-4660-5 (2019).

13. Jones, R. N. Microbial etiologies of hospital-acquired bacterial pneumonia and ventilator-associated bacterial pneumonia. Clin Infect Dis 51 Suppl 1, S81-87, doi:10.1086/653053 (2010).

14. Costa, R. D., Baptista, J. P., Freitas, R. \& Martins, P. J. Hospital-Acquired Pneumonia in a Multipurpose Intensive Care Unit: One-Year Prospective Study. Acta Med Port 32, 746-753, doi:10.20344/amp.11607 (2019).

15. Mohd Sazlly Lim, S., Zainal Abidin, A., Liew, S. M., Roberts, J. A. \& Sime, F. B. The global prevalence of multidrug-resistance among Acinetobacter baumannii causing hospital-acquired and ventilator- 
associated pneumonia and its associated mortality: A systematic review and meta-analysis. $J$ Infect 79, 593-600, doi:10.1016/j.jinf.2019.09.012 (2019).

16. Denis, J. B. et al. Multidrug-resistant Pseudomonas aeruginosa and mortality in mechanically ventilated ICU patients. Am J Infect Contro/ 47, 1059-1064, doi:10.1016/j.ajic.2019.02.030 (2019).

17. Dick, A. W. et al. A decade of investment in infection prevention: a cost-effectiveness analysis. Am J Infect Control 43, 4-9, doi:10.1016/j.ajic.2014.07.014 (2015).

18. Vasudevan, A., Memon, B. I., Mukhopadhyay, A., Li, J. \& Tambyah, P. A. The costs of nosocomial resistant gram negative intensive care unit infections among patients with the systemic inflammatory response syndrome- a propensity matched case control study. Antimicrob Resist Infect Contro/ 4, 3, doi:10.1186/s13756-015-0045-8 (2015).

19. Stewardson, A. J. et al. The health and economic burden of bloodstream infections caused by antimicrobial-susceptible and non-susceptible Enterobacteriaceae and Staphylococcus aureus in European hospitals, 2010 and 2011: a multicentre retrospective cohort study. Eurosurveillance 21 (2016).

\section{Supplementary Files}

This is a list of supplementary files associated with this preprint. Click to download.

- 04PneumoniaMDROsuppletable210822.docx

- 05PneumoniaMDROsupplementmethodresult.docx 